\title{
Neural Networks Based Physical Cell Identity Assignment for Self Organized 3GPP Long Term Evolution
}

\author{
M. Basit Shahab and Abdul Aziz Bhatti
}

\begin{abstract}
This paper proposes neural networks based graph coloring technique to assign Physical Cell Identities throughout the self-organized 3GPP Long Term Evolution Networks. PCIs are allocated such that no two cells in the vicinity of each other or with a common neighbor get the same identity. Efficiency of proposed methodology resides in the fact that minimum number of identities is utilized in the network wise assignment. Simulations are performed on a very large scale network, where initially all the cells are without any PCIs assigned. Results of simulations are demonstrated to analyze the performance of the proposed technique. Discussions about the presence of femto cells and PCI assignment in them are also presented at the end.
\end{abstract}

Keywords - Collision, Confusion, Graph Coloring, Long Term Evolution, Neural Networks, Physical Cell Identity (PCI)

\section{INTRODUCTION}

With an increase in the number of mobile users utilizing cellular networks and thus the wireless technology, demands on systems and their management have increased up to a very high level. Auto configuration, optimization and healing of radio parameters using self-organized networks [1], is a key feature in meeting the requirements of the future mobile cellular networks. Therefore the New Generation Mobile Networks Forum [2] has listed some key parameters that should be handled by adaptive algorithms. 3GPP Long Term Evolution, referred to as LTE and marketed as 4G LTE, is a standard for wireless communication for mobile phones and data terminals. It aims at high data rates [3][4], low delay/latency, high spectral efficiency, high performance broadcast services, cost effective network design, automatic neighbor relation, coverage and capacity optimization, energy saving, interference reduction, physical cell identity automatic configuration, mobility load balancing optimization and random access channel optimization. These goals result in reducing capital expenditures (CAPEX) and operational expenditures (OPEX), by replacing most of the manual work with adaptive techniques that auto tune the network according to the environment changes. One of these parameters is the PCI.

M. Basit Shahab is a Lecturer at Electrical Engineering Department, University of Management and Technology, Lahore, Pakistan (Phone: +92333-9299532; Email: basit.shahab@umt.edu.pk).

Abdul Aziz Bhatti (PhD) is the Dean of School of Science and Technology, University of Management and Technology, Lahore, Pakistan (Phone: +92-305-4440629; Email: drabhatti@umt.edu.pk).

\section{PREVIOUS WORK}

In LTE, each base station named eNodeB broadcasts a distinguishing signal, a fingerprint that helps the user equipment to differentiate between the serving and the neighboring cells [5]. In some circumstances, a conflict occurs among the PCIs of neighboring cells, which results in handover failures when the user equipment being mobile, moves across the boundaries of these regions. This is due to the fact that mobile units can't differentiate between two same broadcasted PCI's, irrespective of their signal strengths. A solution to this problem needs to be explored based on Neighbor cell relation lists [6][7], in order to improve the efficiency of future networks, in the sense that they will have a lesser number of call drops, better quality, improved coverage and efficient mobility handling.

Various types of cases in the PCI assignment issue have been explored [8], according to which it is quite clear that two types of conflicts can occur while assigning the PCIs in a cellular network; when two cells in the vicinity of each other or two cells having a common neighbor are assigned the same PCI. This causes collision in the former, and confusion in the later case. The situations are depicted in Figure 1.

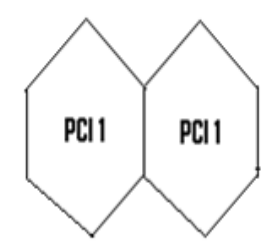

(a)

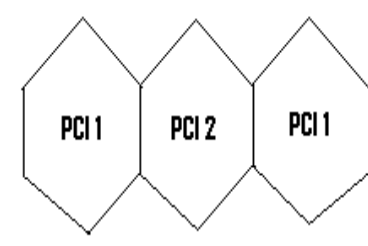

(b)
Figure 1: (a) Collision, (b) Confusion

In the first case; Figure 1 (a), two adjacent cells have the same PCI. When user equipment in either of these two cells moves across the cell boundary towards the adjacent cell with same PCI, a collision is detected and may result in handover failure. In the second case Figure 1 (b), two outer cells having the central cell as a common neighbour have same PCI, resulting in a confusion kind of conflict, which causes handover failure during mobility from either one of them towards the central cell. In order to improvise mobility handling quality throughout the cellular network, these conflicts must be resolved. 


\section{RELATED WORK}

Numerous techniques and algorithms have been proposed to solve these conflicts. Graph coloring is considered as a better approach. In this technique, each cell is considered as a vertex of a graph, and all the cells which are direct neighbours to each other, represented by these vertices are connected through an edge. Trick towards solution is to assign colours to the vertices in such a way, that both cases are properly handled. Solution to Figure 1(a), and 1(b), has been demonstrated in Figure 2(a) and Figure 2(b).

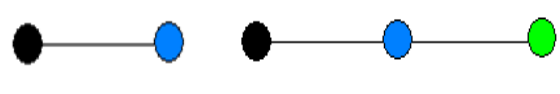

(a)

(b)

Figure 2: (a) Collision free assignment, (b) Confusion free assignment

So far, various graph coloring techniques and methods of graph coloring optimization have been proposed [9] [10] [11]. Graph coloring is used in many applications like VLSI Channel Routing [12], Subcarrier allocation in Cognitive Radio [13], and has been implemented using neural networks [14]. Any graph coloring technique can be applied to solve the PCI assignment task, but the major goal is to resolve the conflicts using minimum number of colours/PCIs. One method, dealing with a four-color-map problem is explored [15], followed by graph coloring using neural networks [16]. A neural network parallel algorithm for channel assignment problems in cellular radio networks is a step that utilizes neural networks in the telecommunication area [17].

\section{MATHEMATICAL MODELLING}

Consider a case of " $n$ " cells in a cellular network and " $m$ " available PCIs or colours to be assigned to these cells with no collision or confusion conflicts. The very famous Hopfield neuron model is considered as shown in Figure 3. Two types of amplifiers are involved in the circuit. An ordinary amplifier and its inverting version are present. Impulse is the input applied to neurons. Feedback paths are also shown. Output is passed through the sigmoid function to determine whether it is 0 or 1 .

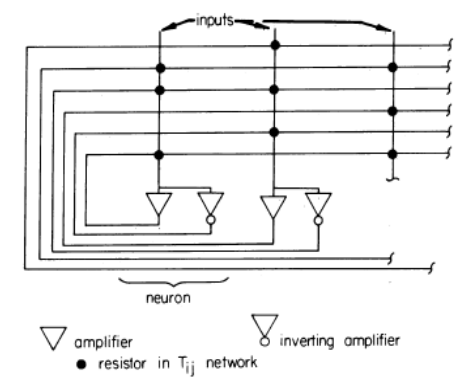

Figure 3: Hopfield neuron model

Three restrictions are applied on the Hopfield network.

1) Inputs and outputs are binary (exclusively ones or zeros). Considering this, it should be quite clear that the nodes produce only binary results.

2) There should be no weights.
3) A condition on the activation function applied such that it is always a unit step.

This model is also applied in artificial neural networks for four-coloring problems and $\mathrm{k}$ colorability [18], an NP complete problem [19], in which colors are assigned to different regions in a map, so that no two regions having a common boundary should get the same color. In four-coloring problem and $\mathrm{k}$ colorability, after all the mathematical work involved, final energy equation for an $\mathrm{i}^{\text {th }}$ neuron in any $\mathrm{X}^{\text {th }}$ region is

$$
\begin{aligned}
E= & \frac{\boldsymbol{A}}{\mathbf{2}} \sum_{X=\mathbf{1}}^{\boldsymbol{n}}\left[\sum_{\boldsymbol{i = 1}}^{\boldsymbol{m}} V_{x i}-1\right]^{\mathbf{2}}+ \\
& B \sum_{X=1}^{n} \sum_{Y=1}^{n} \sum_{i=1}^{m} D_{x y} V_{x i} V_{y i}
\end{aligned}
$$

Where

The first term makes sure that only one neuron's output is 1 in each region. This is done due to the fact that each neurons output corresponds to a particular color. If multiple neurons' outputs are 1 in a particular region, then more than one color will be allocated to this region, which is to be avoided. The second term makes sure that no two adjacent/ neighboring cells get the same color. For any two regions $\mathrm{X}$ and $\mathrm{Y}$, the adjacency matrix Dxy describes the adjacency between these two regions. If $\mathrm{X}$ and $\mathrm{Y}$ are direct neighbors to each other, then the value corresponding to their relationship will be 1 in the matrix Dxy. Thus for this case, if the values of Vxi and Vyi are same which means that both neurons have the same output, there will be an energy penalty equal to the value of the constant $\mathrm{B}$. As this equation always tries to converge to the 0 energy point, therefore, such condition will be avoided. In short, this second term makes sure that the color allocation is collision free.

This is followed by motion equation, which is actually the partial derivative of (1) with respect to neuron's output.

$$
\begin{aligned}
\frac{d U_{x i}}{d t}=- & A\left[\sum_{i=1}^{m} V_{x i}-1\right]- \\
& B \sum_{Y=1}^{n} D_{x y} V_{y i} \sum_{k=1}^{n} D_{y k}+ \\
& C . h\left(\sum_{i=1}^{m} V_{x i}\right)\left(C 1 \sum_{k=1}^{n} D_{x k}+\right. \\
C & \left.\frac{\sum_{k=1}^{n} \sum_{Y=1}^{n} D_{x y} D_{y k}}{\sum_{k=1}^{n} D_{x k}}\right)
\end{aligned}
$$

Colors to the map are allocated by applying sigmoid function on the final motion equation.

$$
\operatorname{Sig}\left(\begin{array}{c}
-A\left[\sum_{i=1}^{m} V_{x i}-1\right]-B \sum_{Y=1}^{n} D_{x y} V_{y i} \sum_{k=1}^{n} D_{y k} \\
+C \cdot h\left(\sum_{i=1}^{m} V_{x i}\right) \\
\left(C 1 \sum_{k=1}^{n} D_{x k}+C 2 \frac{\sum_{k=1}^{n} \sum_{Y=1}^{n} D_{x y} D_{y k}}{\sum_{k=1}^{n} D_{x k}}\right)
\end{array}\right)
$$


This equation is written for the map coloring case, but it can also be used in solving collision free PCI assignment problem. Second case that remains unsolved is the confusion free assignment. This paper extends Yoshiyasu Takefuji's neural networks k-colorability map coloring approach in the form of a single equation for both collision free and the unresolved confusion free PCI assignment. One important factor that needs to be taken care of simultaneously is the upper and lower bounds on chromatic number, which in the other sense means an assignment with minimum number of PCIs utilized.

The proposed technique starts with writing an energy equation like (3) for some $i^{\text {th }} \mathrm{PCI}$ in an $\mathrm{X}^{\text {th }}$ cell. The first thing to be kept in mind is that only one neuron's output should be 1 in any cell, otherwise more than one colors/PCIs will be assigned to a single cell. For that reason, one term in the energy equation, same as the term proposed for collision free assignment, should be present, to deal with the issue. This has been done in (4).

$$
E 1=\frac{A}{2} \sum_{X=1}^{n}\left[\sum_{i=1}^{m} V_{x i}-1\right]^{2}
$$

where A is a constant, used to represent an energy penalty. $\mathrm{V}_{\mathrm{xi}}$ is the output of $\mathrm{i}^{\text {th }}$ neuron in $\mathrm{X}^{\text {th }}$ cell. Second term needs to deal with the case of collision and confusion free assignment, as depicted in (5).

$$
\mathrm{E} 2=B \sum_{X=1}^{n}\left[\sum_{Y=1}^{n} \sum_{Z=1}^{n} \sum_{i=1}^{m} D_{x y} D_{x z} V_{x i} V_{y i} V_{z i}\right],
$$

where $\mathrm{B}$ is a constant that will take the energy away from converging, if a boundary violation takes place. $D_{x y}$ and $D_{x z}$ are elements of the adjacency matrices between $\mathrm{X}, \mathrm{Y}$, and $\mathrm{X}$, $\mathrm{Z}$. $\mathrm{V}_{\mathrm{xi}}, \mathrm{V}_{\mathrm{yi}}$ and $\mathrm{V}_{\mathrm{zi}}$ are outputs of the $\mathrm{i}^{\text {th }}$ neuron in $\mathrm{X}^{\text {th }}, \mathrm{Y}^{\text {th }}$ and $\mathrm{Z}^{\text {th }}$ cell respectively.

$\mathrm{D}_{\mathrm{ij}}=\{1$ if any region $\mathrm{i}$ and $\mathrm{j}$ are adjacent

$\{0$ otherwise

(4) And (5) together result in (6)

$$
\begin{aligned}
E= & \frac{A}{2} \sum_{X=1}^{n}\left[\sum_{i=1}^{m} V_{x i}-1\right]^{2}+ \\
& B \sum_{X=1}^{n}\left[\sum_{Y=1}^{n} \sum_{Z=1}^{n} \sum_{i=1}^{m} D_{x y} D_{x z} V_{x i} V_{y i} V_{z i}\right]
\end{aligned}
$$

Motion equation for an $i^{\text {th }}$ PCI in any $X^{\text {th }}$ cell is the partial derivative of Energy equation with respect to neuron's output

$$
\frac{d U_{x i}}{d t}=-\frac{\partial \mathrm{E}}{\partial V_{x i}}
$$

Because interest lies in the output of some $i^{\text {th }}$ neuron in any $\mathrm{X}^{\text {th }}$ Cell, applying partial derivative on the energy equation with respect to $\mathrm{V}_{\mathrm{xi}}$, results in equation (8).

$$
\begin{aligned}
\frac{d U_{x i}}{d t}=- & \frac{\partial}{\partial V_{x i}}\left[\frac{A}{2} \sum_{X=1}^{n}\left[\sum_{i=1}^{m} V_{x i}-1\right]^{2}\right]- \\
& \frac{\partial}{\partial V_{x i}} B \sum_{X=1}^{n}\left[\sum_{Y=1}^{n} \sum_{Z=1}^{n} \sum_{i=1}^{m} D_{x y} D_{x z} V_{x i} V_{y i} V_{z i}\right]
\end{aligned}
$$

Expanding the $1^{\text {st }}$ term i-e E1 with respect to $\mathrm{X}$ and taking the partial derivative for some specific value, gives

$$
\frac{\partial E 1}{\partial V_{x i}}=A\left[\sum_{i=1}^{m} V_{x i}-1\right]
$$

Writing (5) in terms of $\mathrm{X}$ and $\mathrm{i}$, gives

$$
\begin{aligned}
& \frac{\partial E 2}{\partial V_{x i}}=B \frac{\partial}{\partial V_{x i}}\left[\left[\left[\sum_{Y=1}^{n} \sum_{Z=1}^{n} D_{1 y} D_{1 z} V_{11} V_{y 1} V_{z 1}\right]+\right.\right. \\
& {\left[\sum_{Y=1}^{n} \sum_{Z=1}^{n} D_{1 y} D_{1 z} V_{12} V_{y 2} V_{z 2}\right]+} \\
& \left.\ldots\left[\sum_{Y=1}^{n} \sum_{Z=1}^{n} D_{1 y} D_{1 z} V_{1 m} V_{y m} V_{z m}\right]\right]+ \\
& {\left[\begin{array}{lllll}
\sum_{Y=1}^{n} \sum_{Z=1}^{n} D_{2 y} & D_{2 z} & V_{21} & V_{y 1} V_{z 1}
\end{array}\right]+} \\
& {\left[\begin{array}{lllll}
\sum_{Y=1}^{n} \sum_{Z=1}^{n} D_{2 y} & D_{2 z} & V_{22} & V_{y 2} & V_{z 2}
\end{array}\right]+} \\
& \ldots\left[\begin{array}{llllll}
\sum_{Y=1}^{n} \sum_{Z=1}^{n} D_{2 y} & D_{2 z} & V_{2 m} & V_{y m} & V_{z m}
\end{array}\right]+ \\
& {\left[\left[\sum_{Y=1}^{n} \sum_{Z=1}^{n} D_{3 y} D_{3 z} V_{31} V_{y 1} V_{z 1}\right]+\right.} \\
& {\left[\sum_{Y=1}^{n} \sum_{Z=1}^{n} D_{3 y} D_{3 z} V_{32} V_{y 2} V_{z 2}\right]+} \\
& \left.\ldots\left[\sum_{Y=1}^{n} \sum_{Z=1}^{n} D_{3 y} D_{3 z} V_{3 m} V_{y m} V_{z m}\right]\right]+ \\
& \ldots\left[\left[\sum_{Y=1}^{n} \sum_{Z=1}^{n} D_{n y} D_{n z} V_{n 1} V_{y 1} V_{z 1}\right]+\right. \\
& {\left[\sum_{Y=1}^{n} \sum_{Z=1}^{n} D_{n y} D_{n z} V_{n 2} V_{y 2} V_{z 2}\right]+} \\
& \left.\left.\ldots\left[\sum_{Y=1}^{n} \sum_{Z=1}^{n} D_{n y} D_{n z} V_{n m} V_{y m} V_{z m}\right]\right]\right]
\end{aligned}
$$

Partial derivative is applied for some specific $\mathrm{X}$ and $\mathrm{i}$, but in general, (10) becomes

$$
\frac{\partial E 2}{\partial V_{x i}}=B\left[\sum_{Y=1}^{n} \sum_{Z=1}^{n} D_{x y} D_{x z} V_{y i} V_{z i}\right]
$$

Combining (9) and (11), gives the motion equation

$$
\begin{aligned}
& \frac{\partial E}{\partial V_{x i}}=A\left[\sum_{i=1}^{m} V_{x i}-1\right]+ \\
& B\left[\sum_{Y=1}^{n} \sum_{Z=1}^{n} D_{x y} D_{x z} V_{y i} V_{z i}\right]
\end{aligned}
$$

But $\frac{d U_{x i}}{d t}=-\frac{\partial E}{\partial V_{x i}}$, so

$$
\begin{aligned}
& \frac{\boldsymbol{d} \boldsymbol{U} \boldsymbol{x i}}{\boldsymbol{d} \boldsymbol{t}}=- A\left[\sum_{i=1}^{m} V_{x i}-1\right]- \\
& B\left[\sum_{Y=1}^{n} \sum_{Z=1}^{n} D_{x y} D_{x z} V_{y i} V_{z i}\right]
\end{aligned}
$$

Because this is an NP complete problem, there are many solutions to it. In the line of finding the best one, a hill climbing term needs to be included in the motion equation. Once the hill climbing term has been added, sigmoid function is applied on it. This has been done in (14).

$$
\operatorname{sig}(-P 1-P 2+(P 3 \times P 4))
$$

Where

$$
\begin{aligned}
& \mathrm{P} 1=A\left[\sum_{i=1}^{m} V_{x i}-1\right] \\
& \mathrm{P} 2=B\left[\sum_{Y=1}^{n} \sum_{Z=1}^{n} D_{x y} D_{x z} V_{y i} V_{z i} \sum_{k=1}^{n} D_{y k} D_{z k}\right] \\
& \mathrm{P} 3=C 3 \cdot h\left(\sum_{i=1}^{m} V_{x i}\right) \\
& \mathrm{P} 4=\left(C 1 \sum_{k=1}^{n} D_{x k} C 2 \frac{\sum_{k=1}^{n} \sum_{Y=1}^{n} \sum_{Z=1}^{n} D_{x y} D_{x z} D_{y k} D_{z k}}{\sum_{k=1}^{n} D_{x k}}\right)
\end{aligned}
$$

C1, C2 and C3 are constants, used for normalization $\mathbf{h}\left(\sum_{\mathbf{i}=\mathbf{1}}^{\mathbf{m}} \mathbf{V}_{\mathbf{x i}}\right)$ Is the hill climbing function, which together with normalization constants in the motion equation, forces the state of the system to escape from local minimum and converge to global minimum.

$$
H(x)=\{1 \text { if } x=0
$$

$\{0$ otherwise 
Hill climbing term performs the excitatory force only when all of $\mathrm{V}_{\mathrm{xi}}$ 's are 0 or in other words when PCIs are assigned to all the cells. This means that the algorithm repeats itself unless the best result is achieved.

\section{FEMTOCELLS AND PCI ASSIGNMENT}

The position and number of femtocells are unknown by the operator, and because of the fact that they can be switched on/off or moved at any time by the users; configuration and optimization of a femtocell network cannot be based on classic network design. Secondly, femtocells must be plugand-play devices with a significant degree of selfconfiguration because of non-technical expertise of the femtocells customers. This increases the demand for efficient self-organization techniques for successfully deploying and managing a large femtocell layer over the existing macro cell network. In this way, femtocells are able to react to the changing conditions of the network, traffic and channel, and mitigate cross- and co-layer interference [20]. A lot of studies have been made on femtocells [21] and their efficiency and deployment for providing optimized coverage [22].

Numerous works have been done in providing algorithms and techniques for confusion free PCI assignment in heterogeneous networks. One related work is the extended synchronization signals for eliminating PCI confusions [23]. Distributed PCI assignment in LTE based on consultation mechanism [24], graph coloring based physical cell identity assignment for LTE networks [25] and physical cell identity self-organization for home eNodeBs deployment in LTE [26] are some more famous researches in this area.

The proposed neural networks based technique for PCI assignment can be modified to accommodate the femtocells present inside a macro cell layer. Two basic things need to be taken care of, and the femtocells will be easily configured with PCIs without any confusion:

1. A check for the macro cell's PCI in which the femtocell is present, and then assignment of an extended PCI amongst the extended PCIs available for femtocells.

2. Femtocells within a macro cell needs to be checked for collision or confusion with the femtocells in a particular macro cell, and also with the femtocells of the neighboring macro cell.

More research work is under process to accommodate the femtocells with PCIs using the same but a bit modified neural networks based PCI assignment equations. The new equation needs to have another term which places a condition to check whether the cell considered as a candidate for PCI assignment is a macro or a micro/femto cell, and then assign PCIs to it from the list of available PCIs. The conditions or the new form of equation will simply implement a nested if-else condition (in programming sense), and the task will be achieved.

\section{SIMULATIONS AND RESULTS}

In order to check the performance of proposed technique, a cellular network is considered for assigning Physical Cell Identities. A small part of that network is shown in Figure 4.

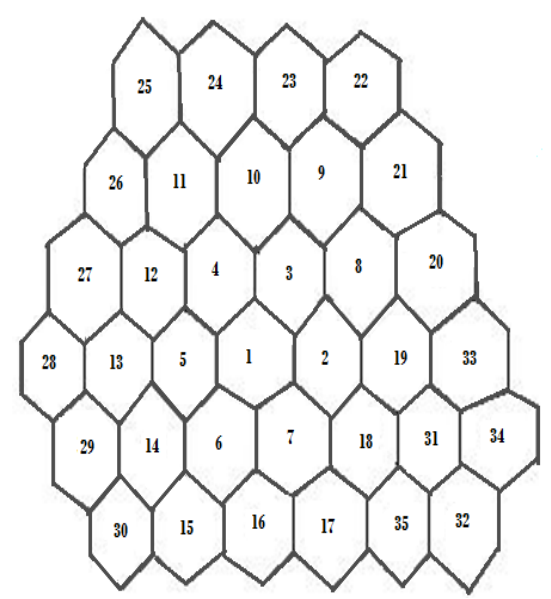

Figure 4: Cellular network model for simulations

Initial simulations are performed for the case of collision free assignment in accordance with equation (3). Results of these simulations describe how the map coloring technique can be used to perform collision free PCI assignment. After that simulations are performed for the proposed technique which accomplishes both the tasks of collision free and confusion free assignment utilizing equation (14). Simulations are performed in following steps.

1. The first step starts with setting values for constants and variables. A, and B being penalty constants are assigned values such that both the terms are optimized. Penalty constants are selected in such a way to vary the impact of constraints for achieving stable results. Hopfield did not propose any proper selection criteria, however, since then multiple strategies have been proposed, among which the one that got great appreciation is Convergence properties of a modified Hopfield-Tank model [27]. This whole strategy was then used to find shortest path in Travelling Salesman problem. The term, which is subjected to more minimization, is multiplied with larger constant and vice versa. Along with penalty constants, ' $m$ ' and ' $n$ ' are also initialized.

2. Second step is to define an adjacency matrix for the cellular system. The adjacency matrix considered for any cell $X$ with all the other cells is given by $D_{x y}$. In actual case, it is present only in the form of a list in the eNodeBs, much easier to manipulate for use in the PCI assignment algorithms.

3. After that, an input vector $U_{x i}$ for all the neurons is defined. $\mathrm{U}_{\mathrm{xi}}$ 's are generated randomly within the range given below initially. It is simply a row vector of order $(1 \mathrm{x} \mathrm{m})$.

$-0.002 \leq \mathrm{U}_{\mathrm{xi}} \leq 0.002$ 
4. Following that is the initialization of the output matrices, and then the implementation of motion equation.

At first, simulations are performed for the map coloring equation (3) based methodology, utilizing it to perform the collision free assignment. A, and B are assigned values 200 and 250 respectively, whereas $\mathrm{C}=\mathrm{C} 1=\mathrm{C} 2=1 . \mathrm{X}, \mathrm{Y}$ and ' $\mathrm{n}$ ' are equal to 35 because they all represent the number of cells. Number of PCIs to be used is calculated using upper and lower bounds on the chromatic number which actually is the minimum number of colors needed to color the graph. 3 PCIs/colors are used to perform proper assignment, thus ' $\mathrm{m}$ ' $=3$. Adjacency matrix $\mathrm{D}_{\mathrm{xy}}$ of order $(35 * 35)$ is defined. After that the motion equation is implemented. All the summations are implemented using loops in the programming. Output of simulations is a matrix $\mathrm{V}_{\mathrm{xi}}$ of order $(3 * 35)$ as shown below.

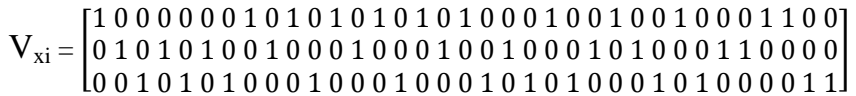

This matrix is the output of an $i^{\text {th }}$ neuron in any $\mathrm{X}^{\text {th }}$ cell. Output array is always an ( $\mathrm{m} \times \mathrm{n}$ ) matrix, with " $\mathrm{m}$ " being the number of colors/PCIs used, and " $n$ " representing the number of cells to which the colors/PCIs are to be assigned. This means the element $V_{3,13}$ of resultant matrix is output of $3^{\text {rd }}$ neuron in the $13^{\text {th }}$ cell. If $3^{\text {rd }}$ neuron represents PCI 3 , then this PCI is assigned to $13^{\text {th }}$ cell if the value of $\mathrm{V}_{3,13}$ in output matrix is 1 . On the basis of values in the matrix Vxi, PCIs have been shown in the corresponding cells in Figure 5, where each number represents a particular PCI and a specific color.

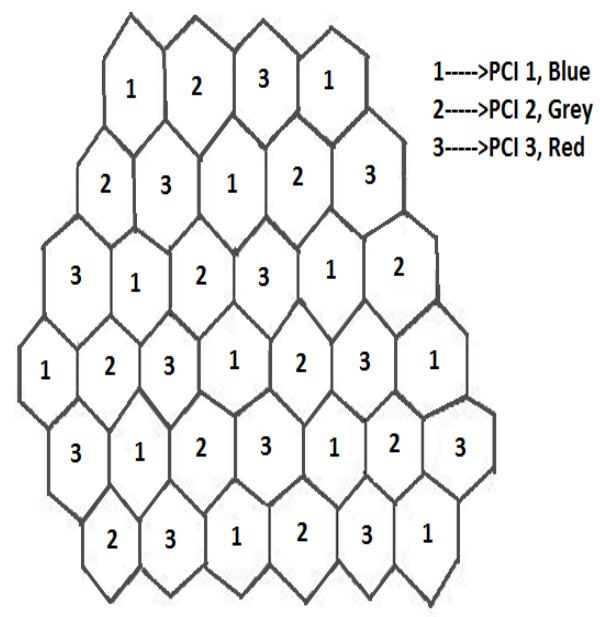

Figure 5: Collision Free PCI Assignment

PCI assignment in figure 5 can easily be analyzed to check whether the map coloring technique is sufficient to provide a good collision free allocation of PCIs or not. It is clearly demonstrated by the figure that no two direct neighbor cells are having the same PCI, thus comfortably achieving the required goal. The numbers 1,2 and 3 actually represent the PCI number, or a specific color in the sense of graph coloring. If 1 stands for PCI 1 or color Blue, then this PCI/color will be assigned to all the cells having number 1 mentioned on them.
The same set of steps is followed for the simulations of proposed technique. $\mathrm{A}, \mathrm{B}, \mathrm{C}, \mathrm{C} 1, \mathrm{C} 2$, and $\mathrm{n}$ are assigned the same values as for the map coloring simulations, but $\mathrm{m}=9$. Because the proposed technique performs both collision and confusion free, another adjacency matrix $\mathrm{D}_{\mathrm{xz}}$ of the order $\left(35^{*} 35\right)$ is defined along with $\mathrm{D}_{\mathrm{xy}}$. This is done because the PCIs of two cells $\mathrm{Y}$ and $\mathrm{Z}$ which are direct neighbors to $\mathrm{X}$ are to be checked for confusion. Result of simulations is a matrix of order $(9 \times 35)$. This matrix is also named $V_{x i}$.

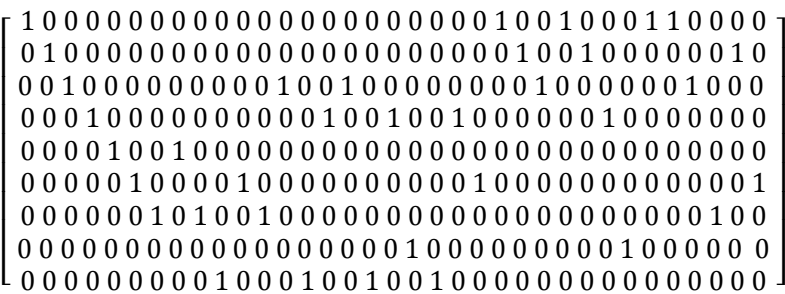

This matrix like the first matrix is the output of an $i^{\text {th }}$ neuron in any $\mathrm{X}^{\text {th }}$ cell. Number of rows is 9 because 9 colors are used to color the considered network with collision and confusion free constraints. Each row contains the output of a particular neuron in all the cells. On the basis of values in the matrix $\mathrm{V}_{\mathrm{xi}}$, PCIs have been shown in the corresponding cells in Figure 6, where each number like the previous case represents a particular PCI and a specific color. If PCI allocation according to the output is considered, only one neuron's output is 1 in each cell, so that no cell has more than one color or PCI assigned to it. This is done by the $1^{\text {st }}$ term of equation (14). The $2^{\text {nd }}$ term results in a collision and confusion free assignment. Moreover $3^{\text {rd }}$ and $4^{\text {th }}$ terms extract the best output out of all the possible solutions. If simulations are performed without the hill climbing function, there can be collision or confusion conflicts at some locations, but still they will be negligible, compared to the number of cells considered for assignment. Also it can result in large number of PCIs/colors utilization for accomplishing the same task that has been done with the minimum number used.
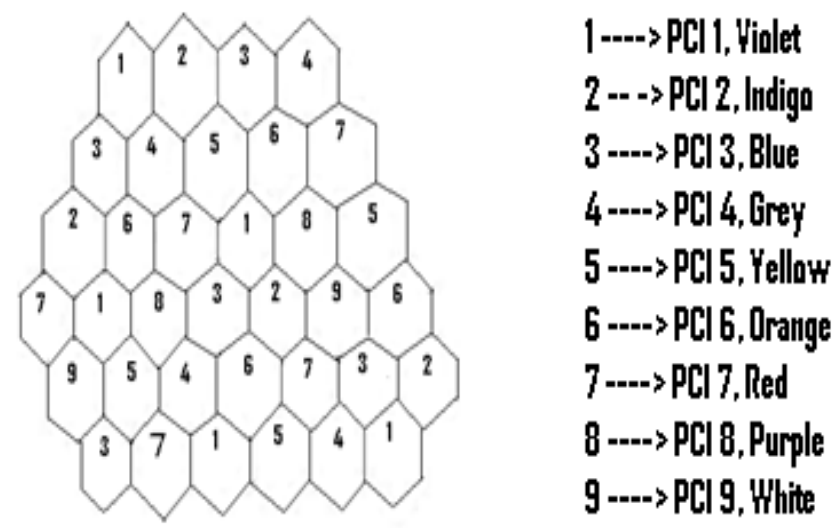

Figure 6: Collision Free PCI Assignment

State of the system always converges to global minimum, supported by almost 750 simulation runs. Figure 7 depicts a 
plot between frequency and number of iteration steps for the proposed technique. Horizontal axis represents the number of iterations required to achieve a satisfactory output, and vertical axis represents frequency; repetition of that particular value of iterations. Reason behind these recursions/iterations is that energy/motion equations are optimization functions, in which different required terms are to be optimized with the help of energy penalty constants. The final output of neurons is the result of these iterations that take place updating values after each iterative step. Optimization is done with the help of constants which offer a penalty increase in energy, if something goes wrong. A lot of recursions take place until these optimization functions converge to the lowest energy value and become stable to give a satisfactory output.

For PCI assignment in each cell, a number of recursions are required to finally find the best assignment, which can be same or different compared to the recursions required for assignment in any other cell. Figure 7 actually depicts the reuse factor of a specific number of iterations during whole network wise assignment for the proposed technique. Average number of iteration steps can be calculated from the graph using any mathematical technique. In this case, average number of iterations is around 980 for about 200 cells.

Simulations were also performed for equation (3); collision free assignment case, for which the average number of iterations were 820 for 200 cells. This difference is because of an additional factor of confusion free assignment along with the collision free case in the motion equation (14), resulting in an increase in recursive steps. If cells in the structure are increased, a corresponding increase in the average number of iteration steps occurs, but this increase is very less, compared to the increase in problem size. This means that the proposed technique is not very much dependent on the problem size.

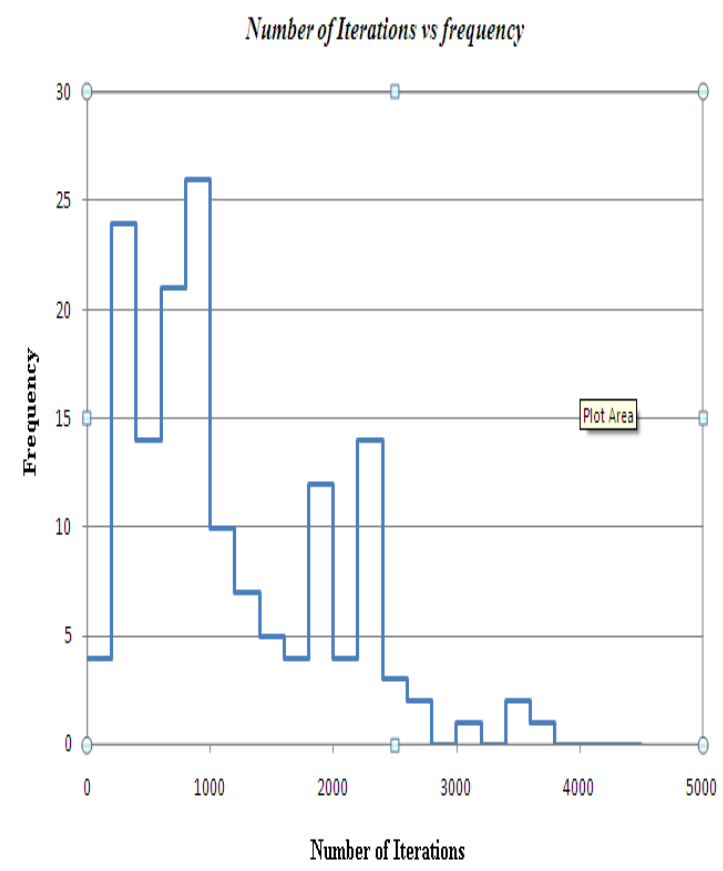

Figure 7: No of iterations and Frequency for Proposed Technique

\section{CONCLUSION}

This paper proposes an algorithm that automatically assigns PCIs to a complete cellular network, during initial network wise configuration, with the least chromatic number [28]. In the same manner, a slight modification in the proposed technique can be used to assign PCIs during incremental growth of an already present network and to accommodate the closed subscriber groups. Another important scenario is the PCI assignment when a conflict takes place. Further improvements to the proposed solution can also resolve PCI conflicts, whenever they occur within a network.

Previous graph coloring techniques solve the collision type of conflict, which mean that they only deal with coloring of adjacent vertices of a graph, and do not deal with assignment of colors without confusion. But in this case both collision and confusion conflicts have been resolved. Secondly neural networks based implementation increases the processing speed because it deals with parallel processing, so the algorithm achieves a better time complexity. Moreover the hill climbing concept improves the algorithm in the sense that the best solution out of all possible ones is extracted at the end, and then it is finally implemented on the whole network.

Having developed and successfully shown the performance of the method, it is believed that the results will have a significant positive impact while dealing with such a problem.

\section{REFERENCES}

1] Sujuan Feng, Eiko Seidel, 2008, "Self-Organizing Networks (SON) in 3GPP Long Term Evolution," Nomor Research GmbH, Munich, Germany.

[2] Hamid Akhavan et al., 2006 "Next Generation Mobile Networks - Beyond HSPA \& EVDO - Whitepaper," Tech. Rep, NGMN Ltd.

E Dahlman, S. Parkvall, J. Sköld, and P. Beming, 2007, "3G Evolution -HSPA and LTE for Mobile Broadband," Elsevier.

3GPP TR 25.913, 2008. "Technical Specification Group Radio Access Network; Requirements for Evolved UTRA (E-UTRA) and Evolved UTRAN (E-UTRAN)," Release 7. Mehdi Amirijoo, Pål Frenger, Fredrik Gunnarsson, Harald Kallin, Johan Moe, Kristina Zetterberg, 2008, "Neighbor Cell Relation List and Physical Cell Identity Self Organization in LTE," Wireless Access Networks,Ericsson Research, Ericsson AB, Sweden.

M. et al and Amirijoo, 2008, "Neighbor cell relation list and measured cell identity management in lte," Network Operations and Management Symposium, NOMS 2008. IEEE.

NGMN, 2007, "Operator Use Cases related to Self Organizing Networks," ver. 1.53.

Tobias Bagndh, Georg Carle, 2009, "Graph Coloring Based Physical-Cell-ID Assignment for LTE Networks,' Proceedings of the International Conference on Wireless Communications and Mobile Computing.

Koljonen, J.-M, 2010, "Distributed generalized graph coloring," IEEE Conference on Self-Adaptive and SelfOrganizing Systems (SASO). 
[10] Mittal, A., 2011, "Graph Coloring with Minimum Colors: An Easy Approach," International Conference on Communication Systems and Network Technologies (CSNT).

[11] Feizi, S., 2010, "Cases where finding the minimum entropy coloring of a characteristic graph is a polynomial time problem," IEEE International Symposium on Information Theory Proceedings (ISIT).

[12] Shenshen $\mathrm{Gu}, 2004$, "A chaotic neural network for the graph coloring problem in VLSI channel routing," International Conference on Communication, Circuits and Systems.

[13] Guobin Zhang, 2010, "Subcarrier allocation algorithms based on graph-coloring in Cognitive Radio NC-OFDM system," IEEE International Conference on Computer Science and Information Technology (ICCSIT).

[14] Di Blas, A. 2002, "Energy function-based approaches to graph coloring," IEEE Transactions on Neural Networks.

[15] Appel K and Haken, 1977, "the solution of the four-colormap problem," Scientific American, pp.108-121.

[16] Philipsen, W.J.M.; Stok, L, 1991 "graph coloring using neural networks," IEEE International Symposium on circuits and systems.

[17] Funabiki, N, 1992, "A neural network parallel algorithm for channel assignment problems in cellular radio networks," IEEE transactions on Vehicular technology.

[18] Takefuji Y and Lee K. C, 1991, "Artificial neural networks for four-coloring problems and k colorability problems," IEEE Trans, on Circuits and Systems, 38, 3, pp.326-333.

[19] Garey M.R., and Johnsan D.S., (1979), Computers and Intractability: A Guide to the Theory of NP-Completeness. (W.H. Freeman and Company).

[20] Ladányi, A. ; López-Pérez, D. ; Jie Zhang, 2012, "Selforganization for LTE enterprise femtocells," GLOBECOM
Workshops
6-10
Dec,
2012.

[21] H. Claussen, L. T. W. Ho, and L. G. Samuel, "An overview of the femtocell concept," Bell Labs Technical Journal, vol. 3, no. 1, pp. 221-245, May 2008.

[22] H. Claussen, L. T. W. Ho, and L. G. Samuel, "Selfoptimization coverage for femtocell deployments," in Wireless Telecommunications Symposium, ser. 24-26, California, USA, April 2008, pp. 278-285.

[23] Zahran, A.H., 2012, "Extended synchronization signals for eliminating PCI confusion in heterogeneous LTE," WCNC 1-4 April 2012.

[24] Y. Liu, W. Li, H. Zhang, and L. Yu, "Distributed PCI Assignment in LTE Based on Consultation Mechanism," in Proc. 6th Int Wireless Communications Networking and Mobile Computing WiCOM Conf, pp. 1-4, 2010.

[25] T. Bandh, G. Carle, and H. Sanneck, "Graph coloring based physical-cell- ID assignment for LTE networks," in Proc. of the 2009 Intl Conf. on Wireless Communications and Mobile Computing, IWCMC '09, pp. 116-120, New York, NY, USA: ACM, 2009

[26] Y. Wu, H. Jiang, Y. Wu, and D. Zhang, "Physical Cell Identity Self- Organization for Home eNodeB Deployment in LTE," in Proc. 6th Int Wireless Communications Networking and Mobile Computing (WiCOM) Conf, pp. $1-6,2010$.

[27] Bizzarri AR, 1991," Convergence properties of a modified Hopfield-Tank model," Biological Cybernetics, volume 64, number 4

[28] Sen, A.; Roxborough, T.; Medidi, S, 1998, "Upper and lower bounds of a class of channel assignment problems in cellular networks," Seventeenth Annual Joint Conference of the IEEE Computer and Communications Societies, Proceedings, IEEE. 